\title{
i
}

\section{La commutazione di codice nella classe di lingua: prime considerazioni Roberta Ferroni}

ABSTRACT: In questo articolo saranno analizzate alcune delle funzioni svolte dall'alternanza di codice dentro un contesto di insegnamento dell' italiano come lingua straniera. Tenteremo inoltre di stabilire se il livello di apprendimento della $\mathrm{L}$ s e certe attività didattiche possono influenzare tale fenomeno. PAROLE CHLAVE: insegnamento di lingua straniera; alternanza di codice; mezzi di comunicazione; lingua italiana.

\section{Introduzione}

L'apprendimento/insegnamento di una lingua straniera si differenzia dalle altre situazioni educative per una ripartizione dei ruoli particolarmente asimmetrica; generalmente l'insegnante di Ls ha una competenza linguistica superiore a quella dei suoi allievi e questo lo porta ad occupare un tempo maggiore in termini di parole. Inoltre ciò che si insegna e si apprende è contemporaneamente il mezzo con il quale si insegna e quello con cui si apprende. Questo fenomeno che Bange chiama di bifocalizzazione fa si che gran parte degli apprendenti focalizzi la propria attenzione sulla forma piuttosto che sui contenuti:

[...] il focus dell'attenzione degli allievi si trova di fatto portato verso la lingua, verso i mezzi di comunicazione e non verso i suoi contenuti. La bifocalizzazione propria della comunicazione esolingue cambia di natura: c'è una focalizzazione sulla lingua, l'obietrivo tematico della comunicazione è gettato alla periferia". (Bange, 1992: 73) 
Da questa identità tra oggetto di studio e strumento di studio derivano comportamenti verbali che si realizzano come "rituali" comunicativi, non obbligati ma ricorrenti (Cicurel, 1990). La commutazione di codice rientra fra tali rituali e si manifesta nella classe di lingua come strategia per superare queste difficoltà.

Breve quadro tcorico

Le ricerche effettuate fino ad ora sulla commutazione si sono concentrate soprattutto su classi bilingui in situazioni di immersione (Moore, 1996); solo di recente si è assistito ad un allargamento del campo d'indagine alla classe di lingua straniera, dove lo studio dell'alternanza porta a considerazioni ben differenti ${ }^{1}$

Molti dei lavori pubblicati hanno indagato, partendo da una prospettiva sociologica e sociolinguistica, le cause e i fattori alla base di questo processo, fra di essi: i livelli di conoscenza della Ls dei discenti (Causa, 1997); la tipologia delle attività (Castellotti, 1997); il progetto pedagogico dell'insegnante (Garbédian, Lerasle 1997). Un'osservazione che emerge da molti di questi studi è che non esiste un rapporto univoco fra tipo di classe di lingua e modalità di commutazione, anche se risulta possibile rintracciare delle costanti che operano in determinati contesti.

Partendo da questi studi, analizzeremo i cambi di codice effettuati nella classe di lingua italiana, tentando di applicare le due grandi categorie proposte da Cambra e Nussbaum (1997). La prima categoria è quella che loro chiamano commutazione, composta cioè da tutti quei cambi con valore comunicativo; l'altro grande gruppo è detto delle alternanze ed è formato dai passaggi dalla Ls alla $\mathrm{Lm}$ al fine di rendere l'imput comprensibile: sono alternanze con funzione esplicativa e vengono effettuate per mezzo di riformulazioni e traduzioni letterali. Lo studio prenderà principalmente in esame i cambi degli insegnanti.

1. Si veda "Alternance des langues et apprentissages", in Écudes de Linguistique Appliquée (1997), interamente dedicata all'alternanza di codice in classi di lingua straniera. 


\section{Descrizione del corpus e metodologia}

Il nostro studio si fonda su un corpus composto da 14 ore di registrazione effertuate presso i corsi di lingua italiana della USP ${ }^{2}$, nei livelli elementari ed avanzati. Dei quattro professori osservati uno è madrelingua, mentre gli altri sono brasiliani e hanno appreso l'italiano come lingua straniera. Gli studenti hanno un'età compresa fra i 30 e i 60 anni.

Prima di procedere all'analisi è opportuno precisare che i risultati forniti da questa ricerca sono puramente descrittivi, pertanto non siamo ancora in grado di trarne delle conclusioni di carattere né teorico né didattico ${ }^{3}$.

\section{Cli obicttivi}

Dato che sembra ormai superato il mito che vorrebbe la classe di lingua come luogo di iterazione esclusivamente in lingua straniera, oggetto d'interesse della nostra ricerca sarà uno studio da un punto di vista microdidattico dell'alternanza di codice tra Lm e Ls, dentro un contesto di insegnamento dell'italiano come lingua straniera. A partire dagli esempi selezionati, lasciando da parte la natura intrinsecamente linguistica di questo fenomeno, analizzeremo:

- il ruolo e alcune delle funzioni che questa pratica riveste;

- se certe attività didattiche possono indurla o inibirla;

- se il livello di apprendimento della Ls può influire sul processo;

- se i passaggi dall'una all'altra lingua sono anticipati da marcatori.

2. Sono i cosiddetri corsi di italiano nel Campus.

3. Questa prima analisi fa parte di un progetto di ricerca in corso. 
L'alternanza con valore regolativo e metalinguistico

Da una prima ricognizione dei dati si può constatare che i cambi di lingua sono iniziati con maggiore frequenza dagli studenti ${ }^{4}$, tuttavia nel corpus sono piuttosto frequenti casi in cui l'iniziatore è l'insegnante, anche se come è stato notato "l'insegnante di lingua che ricorre alla lingua materna in classe di lingua straniera lo fa il più delle volte con un senso di cattiva coscienza" (Coste, 1997: 395).

In questa prima parte cercheremo di descrivere e classificare i cambi degli insegnanti che appartengono alla categoria dei cosiddetti cambi di commutazione (Cambra, Nussbaum, 1997).

In primo luogo, per gestire le attività di classe, la Ls viene usata per istruzioni routinarie, mentre l'insegnante ricorre alla $\mathrm{Lm}$, nei livelli elementari, quando deve dare istruzioni più complesse (ad esempio i giochi). Si consideri l'esempio 1:

\section{Esempio 1}

I Avete quest'altra attività prima di iniziare l'unità quattro, va bene?

C Si.

I Allora mettete via questo: (togliendo dal banco di uno studente un'altra attività) e distribuite le carte, va bene? Il gioco si chiama l'INTRUSO + L'INTRUSO è come il MICO + + (una studentessa cerca di prendere la parola). Cosa T.?

A (??)

I Não (+) prestem atenção pra depois não ficar perguntando (+) tá? Então o jogo é assim + è o IM:: SI CHIAMA l'intruso + CHI è l'intruso? É o mico né? Quem que está com ele no fim (+) perde!

\section{A Quem atrapalha}

I È (+) allora c'è sempre una domanda e una risposta "la camera è tranquilla?" "Sì (+) dà sul cortile interno" (leggendo le carte) quindi si devono abbinare le

4. Questo dato è confermato anche da numerosi studi (Castelletti, Moore, 1997). 
domande $(++$ ) alle risposte (rivolgendosi alla stessa studentessa che sembra non aver capito) tem de sempre juntar a pergunta com a resposta, formar uma dupla e aí (+) uma comprando do outro, tá? Quem ficar com o intruso no fim perde

A Sono io o:: mico (risate)

L'esempio è tratto da un livello elementare, scopo del gioco era di abbinare domande a risposte. Inizialmente l'insegnante aveva diviso la classe in due gruppi e dato la consegna in italiano. Siccome non tutti sembrano aver capito le istruzioni, l'insegnante tenta nuovamente, questa volta però per evitare malintesi ricorre ad una traduzione immediata "l'INTRUSO è come il MICO", nella sequenza successiva richiama lo studente distratto, spostando il proprio codice "Não (+) prestem atencão..." In questo modo l'insegnante mette in atto contemporaneamente due strategie: prima di tutto si assicura l'attenzione del gruppo, dall'altro, spiegando le regole in portoghese, evita di fare un grosso lavoro in italiano che richiederebbe molto più tempo e possibili fraintendimenti. C'è poi la tendenza da parte degli insegnanti a rivolgersi in Ls nelle iterazioni con tutta la classe e in portoghese con i singoli studenti. Nello stesso estratto l'insegnante passa al portoghese ogni volta che lo studente fa cenno di non capire.

Per affrontare riflessioni o commenti di carartere metalinguistico gli studenti sia del livello elementare che avanzato preferiscono ricorrere alla $\mathrm{Lm}$, così che la $\mathrm{Ls}$ finisce per svolgere la funzione di lingua-oggetto, "cioè viene usata in citazioni volte ad illustrare le caratteristiche del sistema linguistico oggetto di studio" (Anderson, 2003: 83). Si veda nell'esempio seguente dove il sintagma "L'abbigliamento" assume questo valore:

\section{Esempio 2}

A Pode por L'abbigliamento, o é masculino

I No:::elle apostrofo è l'articolo per (+) maschile e femminile... abbigliamento è maschile è una parola maschile 
Diversa è la modalità degli insegnanti che per costruire una riflessione sulla lingua straniera usano varie strategie (semplificazioni, ripetizioni, parafrasi). Solo se gli allievi continuano a manifestare difficoltà di comprensione, che sono più che mai di carattere terminologico, allora la lingua madre s'impone negli interventi dell'insegnante:

\section{Esempio 3}

I Il condizionale è un modo anche in :: portoghese (+) e come si chiama?

\section{A Pretérito?}

I No::: questo è il congiuntivo! Il condizionale noi lo chiamiamo com'è in portoghese, + + futuro do + [PRETÉRITO]

\section{C [PRETÉRITO]}

I No? (rumori) In portoghese (++) EU FARIA, que eu faria? O tenho ou que eu teria feito ci sono questi due (+) no?

A (??)

I E il tempo composto futuro do pretérito

In questa sequenza l'insegnante ricorre alla Lm solo per fare un po' di ordine nella nomenclatura; questo tipo di alternanza non innesca una riflessione in termini metalinguistici, quanto piuttosto richiama ad una traduzione terminologica, visto che entrambe le lingue condividono il modo condizionale.

L'insegnante ricorre, dunque, al portoghese per dare chiarimenti di ordine culturale, cioè quando deve spiegare un'usanza o un elemento italiano, come nel seguente caso:

\section{Esempio 4}

A Devono: pagare un supplemento:

I Un supplemento rapido (+) NO? Perché?

A Per il cibo?

I NO!... è una tassa che paga chi usa i treni: ci sono gli intercity no? Gli intercity 
:::sono dei treni che vanno:: fuori Italia a più alta velocità não è uma taxa de embarque:: seria como o TGV

In questo esempio il passaggio al portoghese può essere interpretato come un rentativo dell'insegnante di trasferire un aspetto culturale tipicamente italiano a parlanti stranieri che non lo conoscono.

L'alternanza come supporto alla comprensionc: le traduzioni, i segni d'incomprensionc o verifica di senso

Dal nostro corpus risulta che una delle funzioni principali svolte dall'alternanza è quella di collaborare per la costruzione di un senso in Ls. Sia gli insegnanti che gli studenti ricorrono a tali operazioni in modo che la lingua dominante può cambiare rapidamente da un passaggio all'altro.

Fanno parte di questa categoria tutte quelle forme di passaggio dalla $\mathrm{Ls}$ alla $\mathrm{Lm}$ che servono per rendere l'imput comprensibile. Questi cambi, denominati alternanze (Cambra, Nussbaum, 1997), hanno una funzione esplicativa o preventiva e sono realizzati per mezzo di traduzioni letterali che si situano all'interno di un turno di parola.

Il corpus contiene moltissimi di questi esempi. Spesso gli studenti sono gli iniziatori, ma in certi casi è l'insegnante stesso che ricorre alla traduzione, specie quando la difficoltà sussiste. Il seguente estratto ne è un esempio:

\section{Esempio 5}

A Spazz::olando (??)

I SPAZZOLARE (+) NO! Spazzolare i capelli o spazzolare i denti (imitando le azioni)

A (fa cenno di no con la testa)

I SPAZZOLARE $(0,3)$ escovar e pentear!

A Ah:: qual é escovar? 


\section{Spazzolare!}

Alla richiesta di aiuto di uno studente, l'insegnante prima interviene imitando le azioni, poi siccome l'incomprensione continua, ripete con enfasi la parola in italiano e alla fine la traduce in $\mathrm{Lm}$.

La traduzione funziona anche, da parte dei professori, come mezzo per confermare la comprensione e per riportare il cambio verso l'impiego della Ls:

\section{Esempio 6}

I Guardate questi disegni (+) andare diritto (+) che cos'è andare diritto?

\section{Al Reto}

\section{A2 Reto é isso?}

I E andare dritto

Così il professore può utilizzare la traduzione immediata di una parola, allorché la difficoltà appaia evidente da parte della classe. Nel passaggio seguente, l'insegnante, per sottolineare l'importanza della parola "pantano" usa un accento intenso, dopodiché la scrive alla lavagna e fa una traduzione immediata:

\section{Esempio 7}

I Allora la rana dalla bocca larga $(++)$ viveva in uno STAGNO:: $(++)$ pantàno/ pântano scrivendo alla lavagna stagno)

Come si passa da una lingua all'alıra

Come avviene il passaggio da un codice all'altro? Esistono dei marcatori che anticipano questo passaggio?

Un primo dato che emerge è che il passaggio dal portoghese all'italiano avviene nel modo più naturale possibile, mentre l'alternanza tra italiano-portoghese è spesso anticipata da marcatori. 


\section{Esempio 8}

A In pratica questo testo tratta eh::: di un:: casal

In questo caso uno studente manifesta la sua incapacità di continuare il discorso iniziato in italiano. Studenti e professori legittimano i loro passaggi al portoghese per mezzo di allungamenti vocalici, ripetizioni e impossibilità di terminare una frase. Ma l'insegnante possiede anche altre strategie per passare al portoghese, specialmente quando dà spiegazioni, o riformulazioni in funzione metalinguistica:

\section{Esempio 9}

I DOVETE formare le coppie + + la domanda con la risposta dovete abbinare allora per esempio (prendendo le carte e leggendo) "La camera è tranquilla? Sİ:: dà sul cortile interno"

non è una domanda e una risposta::: allora chi ha questa domanda e questa risposta deve abbassare (abbassando le carte) abbassarle e poi per formarne altre, dovrà comprare le carte dal compagno capito? É igual au jogo do mico + que a gente joga

Il passaggio alla lingua portoghese è annunciato da "capito?" L'utilizzo di questo termine sollecita un effetto di ritorno da parte degli allievi che sarà immediatamente confermato da una traduzione.

In altri casi, l'insegnante usa delle strategie dirette per invitare la classe alla commutazione; per tale scopo si serve di modalità interrogative basate sulla traduzione o sulla spiegazione di una parola, come in questi esempi:

\section{Esempio 10}

I ...Dove mettiamo il riso? Sai cos'è il riso? Cos'è il riso ragazzi?

\section{Arroz}


La domanda rivolta alla classe innesca un cambio di lingua, una traduzione. Altre volte addirittura ricorre a modalità ancora più esplicite, un esempio tipico è il seguente:

\section{Esempio 11}

I Ragazzi è UGUALE al portoghese UGUALE non è difficile è uguale come lo traduco questo qua? (indicando la frase scritta alla lavagna)

\section{AACada ano}

Infine, l'insegnante invita gli srudenti ad alternare le lingue specie dal portoghese all'italiano, questi passaggi si manifestano quasi sempre in contesti comunicativi. Lo può fare come nell'esempio 12 invitando lo studente che aveva iniziato una sequenza in portoghese a parlare in italiano "no no in italiano" o (esempio 13) riprendendo lui stesso il discorso in Ls "Ah:::è già già hai ragione":

\section{Esempio 12}

I Chi continua? L. P. M.?

\section{A Eu não sei falar (rumori)}

I No no in italiano in italiano piano piano... Esempio 13

I ... Chi va in albergo da solo?

\section{Al Il signor Croci}

\section{Perché?}

Al Perché::::vuole prenotare una camera singola

I = Bravissima

\section{A2 Mais o outro também + ele também vai:::}

I Ah:::.è già già hai ragione (??) anche Nillo + Nillo e Croci vanno da soli in albergo 


\section{Prime conclusioni}

Da una prima analisi, possiamo constatare che il fenomeno da noi osservato si manifesta in maniera eterogenea da una classe all'altra, da un livello all'altro, da un insegnante all'altro. S'interroga a proposito Castellotti (2001), che attribuisce questa discordanza ad alcuni fattori: il grado di comperenza degli allievi; la natura delle attività; le scelte e lo stile pedagogico dell'insegnante e la competenza linguistica di quest'ultimo.

Tuttavia, alla luce dei dati raccolti, possiamo già formulare alcune ipotesi sulla natura e la funzione dei cambi di lingua studiati fino a questo momento.

In primo luogo possiamo tentare di fare una suddivisione secondo i seguenti criteri:

- Cambi di senso: comprendono turti quei passaggi che hanno come scopo primario quello di rendere l'imput comprensibile e si realizzano per mezzo di traduzioni, specie di unità lessicali. Attualmente queste risultano essere fra le più numerose e diffuse su tutti i livelli.

- Cambi di gestione: in essi, in generale, la Lm viene usata per istruzioni che presentano un più alto livello di complessità (come ad esempio i giochi); questi passaggi sono particolarmente produttivi nei livelli elementari.

- Cambi metalinguistici: comprendono quegli spostamenti in cui si riflette sul funzionamento della lingua; questo tipo di commutazione, comune a turti i livelli, è più che mai di carattere terminologico, visto l'alto grado di parentela fra le due lingue.

Sebbene i risultati siano ancora parziali, possiamo dire che, in gran parte dei casi, gli apprendenti sono gli iniziatori dei cambi.

Inoltre, contrariamente a quanto si possa immaginare, l'alternanza è prodotta in maniera abbastanza frequente anche da apprendenti con una competenza maggiore; questo dipende molto dal tipo di attività che si sta svolgendo in classe. Nella 
maggioranza dei casi osservati, le commutazioni coincidono con attività di tipo orale che richiedono uno sforzo maggiore.

Dato che questo lavoro fa parte di una progetto di ricerca in corso, ci auguriamo che ricerche successive possano ampliare le informazioni fin qui ottenute.

RESUMO: Neste artigo sāo analisadas algumas das funçōes da alternància de código num contexto de ensino de italiano como lingua estrangeira. Pretende-se, além disso, estabelecer se o nivel de aprendizagem da $L$ s e algumas atividades didáticas podem influenciar esse fenômeno.

PALAVRAS-CHAVES: ensino de lingua estrangeira; alternância de código; meios de comunicą̧äo; lingua italiana.

\section{Bibliografia}

ANDERSON L. (2003), "Processi di commutazione di codice nella classe di lingua". In CILIBERTI, PUGLIESE, ANDERSON, Le lingue in classe. Carocci, Roma, pp. 75-91.

BANGE P. (1992), “À propos de la communication et de l'apprentissage de L2 (notamment dans ses formes istitutionnelles)". AILE, 1, pp. 53-85.

CAMBRA M., NUSSBAUM L., (1997), "Gestion des langues en classe de LE. Le poids des représentations de l'enseignant". Études de Linguistique Appliquée, 108, pp. 423-32.

CASTELLOTTI V. (1997), "Langue érrangère et français en milieu scolaire: didactiser l'alternance?" Érudes de Linguistique Appliquée, 108, pp. 401-10. (2001), La langue maternelle en classe de langue étrangère. CLE International, Paris.

COSTE D. (1997), “Alternance didactiques". Études de Linguistique Appliquée, 108, pp. 393-400. DABÈNE L., CICUREL F., LAUGA-HAMID M.-C., FOERSTER C. (éds.) (1990), Variations et rituals en classe de langue. Hatier-Credif, Paris.

Norme di trascrizione

I insegnante

A allievo

AA almeno due allievi 


\section{C gruppo-classe}

(?testo?) trascrizione incerta

(??) testo del tutro incomprensibile

... porzione di testo omesso

(commento) commento sul comportamento non verbale

$(+)$ pausa breve (inferire a un secondo)

(++) pausa più lunga (inferiore a un secondo)

(n) pausa lunga ( $\mathrm{n}$ = lunghezza in secondi)

= allacciato al turno immediatamente precedente

[testo] due turni di parola in sovrapposizione

[resto]

TESTO a voce alta, o con accento marcato

testo elementi rappresentativi del fenomeno analizzato

testo: suono allungato (il numero di : indica il grado di allungamento)

testo- gruppo tonale interrotto

intonazione discendente

, intonazione sospensiva

? intonazione ascendente

! intonazione discendente

NB: I nomi di persona sono stati abbreviati per mantenere l'anonimato. 